\title{
Design of a Multimodal Light Sheet Optical System for Multivariate Applications in Phytopathology
}

\author{
Mama Sangare ${ }^{1 *}$, Mathieu Hebert ${ }^{2}$, Anthony Cazier ${ }^{2}$, Pierre Chavel ${ }^{2}$ \\ ${ }^{1}$ Institut des Sciences Appliquées, Université des Sciences Techniques et Technologies de Bamako (USTTB), Bamako, Mali \\ ${ }^{2}$ Laboratoire Hubert Curien, Institut d’Optique Graduate School, Université Jean Monnet de Saint-Etienne, Université de Lyon, \\ CNRS, Saint-Etienne, France \\ Email: *mamasangare101186@gmail.com
}

How to cite this paper: Sangare, M., Hebert, M., Cazier, A. and Chavel, P. (2021) Design of a Multimodal Light Sheet Optical System for Multivariate Applications in Phytopathology. Optics and Photonics Journal, 11, 110-120

https://doi.org/10.4236/opj.2021.115009

Received: March 29, 2021

Accepted: May 5, 2021

Published: May 8, 2021

Copyright (C) 2021 by author(s) and Scientific Research Publishing Inc. This work is licensed under the Creative Commons Attribution International License (CC BY 4.0).

http://creativecommons.org/licenses/by/4.0/

\begin{abstract}
The design of optical instruments is an active subject due to improvement in lens techniques, fabrication technology, and data handling capacity. Much remains to do to expand its application to phytopathology, which would be in particular quite useful to improve crop growth monitoring in countries like Mali. An optical multimodal system for plant samples has been developed to improve the characterization of leaf disease symptoms, provide information on their effects, and avoid their spread. Potentially inexpensive components (laser, lens, turntables camera and sample, filter, lens, camera and computer) have been selected, assembled and aligned on an optical table into a multimodal system operating in transmission, reflection, diffusion and fluorescence. The illumination and observation angles can be adjusted to optimize viewing conditions in the four modes. This scientific contribution has been an initiation into the design and implementation of an optical instrument. Initial results are shown and will now be extended in cooperation with agronomic laboratories in African countries for tests on specific plant diseases in relation with prevailing climate conditions.
\end{abstract}

\section{Keywords}

Design, Optical System, Light Sheet, Multimodal, Multivariate, Phytopathology

\section{Introduction}

Optical spectroscopy since its emergence as an essential analytical technique is a rapidly expanding field, and its applications range from medical diagnostics to 
the environment to agriculture. However, the penetration of the technique in the disadvantaged regions, where the needs are very numerous, comes up against the challenge of developing robust and economical solutions. This may be due to ignorance; theory, the operating principle of an optical spectroscope equipment, the use of this analysis technique in the fields of applications or the lack of exchanges between scientific research laboratories and Malian economic communities as well than those elsewhere. This article will focus on the application to agriculture and on countries whose economies need special support. By elsewhere, studies have been carried out at Lund University in Sweden to design a multimodal optical system; which can be used in Transmission, Reflection, Diffusion and/or Fluorescence mode by modification of a conventional optical microscope. Its operating principle consists to illuminate objects using 13 lightemitting diodes to acquire multispectral and multimodal images ranging from ultra-violet to near infrared [1] [2]. The multimodal microscope has been exploited everywhere by physics scientists in West and Central Africa; while developing powerful techniques for wide multidisciplinary and multivariate application in the health field to diagnose malaria [3]-[10]. Even if, its application was initially limited to the study of malaria, the use of the optical and multimodal microscope in the field of agriculture has also been the subject of numerous studies and publications by physicists in Mali and in Côte d'Ivoire by developing multispectral imaging and multivariate analysis techniques in order to understand in real time the epidemiology of certain pathogens to help biologists, biochemists and agronomists in their fight against pathogens [11] [12] [13] [14]. As such, optical and multimodal spectroscopy has been a complementary study of the preventive measures adopted by these researchers evolving in phytopathology for the eradication of these devastators [15]-[20]. It is likely that, this tool was conceived by certain elements of optics; however, it was not modifiable to perform fluorescence and polarization studies. It was used only for the studies in transmission, reflection and diffusion. Another research was carried out on multispectral to develop the multimodal imaging measurement instrumentation protocol studying wavelength by wavelength each of the light-emitting diode modules [21]. In addition, the University of Angers in partnership with the Institut d'Optique Graduate School of the Jean Monnet University in Saint-Etienne has produced an experimental model of a 3D light sheet microscope whose operating principle uses two Lasers for the study of fluorescence in biology by light scattering [22].

In light of Rousseau's innovation in 2017, an optical system has been put in place for multimodal studies in transmission, reflection, scattering and fluorescence. It can be used particularly in the field of phytopathology in order to detect any parasitic infection on cultivated leaf plants. The device has a principle of operation also with laser lighting and/or LED (Light Emitting Diode) for the microscopic observation of biological samples through spatial information in order to carry out their spectroscopic and multimodal detection study. The relevance of this work for agriculture in regions with modest resources mainly contributes 
to the development of the potential of the innovated instrument and its uses at different scales in phytopathology; able to solve spectral diagnostic problems studying the effect of bacteria, viruses or other types of chemical or biological elements on plants.

\section{Materials and Methods}

The system demonstrated is composed of the following elements.

- A laser diode (635 $\mathrm{nm}$ class II) for the illumination of the sample coupled to a lens (spherical or cylindrical according to the needs of the experiment) of focal length $f=175 \mathrm{~mm}$ and at a linear optical density to adjust the level lighting.

- A $435 \mathrm{~nm}$ high-pass filter (435FG03-25-Andover) cuts all wavelengths below $435 \mathrm{~nm}$ and a $405 \mathrm{~nm}$ laser making it possible to emit only the fluorescence emission at a length of precise wave.

- A 1/3 $1280 \times 1024$ USB CMOS color camera (DCC1645C-Thorlabs) coupled with a Canon EF-S $60 \mathrm{~mm} \mathrm{f/2.8} \mathrm{macro} \mathrm{USM} \mathrm{lens.}$

- Newport Micro-control opto-mechanical elements including all the supports, the fixings and the two turntables of rotation for the sample and the camera: M-UTR80 and M-UTR120, these two turntables of rotation being necessary for the choice of the study modes in transmission, reflection, diffusion and fluorescence.

As such, the optical system is appropriate for the analysis of samples of millimeter size (See diagrams of the optical system in the "Results" section).

Each of the study modes cited corresponds to a choice of lighting and observation directions. The camera's axis is the direction of observation and this last is placed in the plane of incidence, defined by the normal to the sample and the direction of illumination. By designating by $\theta$ the angle of incidence of the illumination beam on the sample and by $\alpha$ the angle made, in the direction of light, the axis of the illumination beam with the axis of the camera (see Figure 1).

In transmission mode, the sample is illuminated normally and the axis of the camera is in the lighting extension, so that it is illustrated in Figure 1, therefore $\alpha=0^{\circ}, \theta=0^{\circ}$.

In reflection mode, it is important to exploit the variation in reflectance as a function of the angle of incidence. By following specular and non-diffuse reflection, Descartes' law for reflection involves:

$$
\alpha=\pi-2 \theta
$$

For example, if the angle of incidence on the sample is $50^{\circ}$ (relative to the source), the reflected signal picked up by the camera is $80^{\circ}$.

In diffusion and fluorescence, the sample may be illuminated at an angle of $45^{\circ}$ with the camera being kept normal to the sample, so $\theta=45^{\circ}, \alpha=90^{\circ}$.

The following Table 1 summarizes the test angle selected.

By respecting these choices for each mode, its operating principle is similar to that of the conventional microscope, or more exactly to an assembly macro 


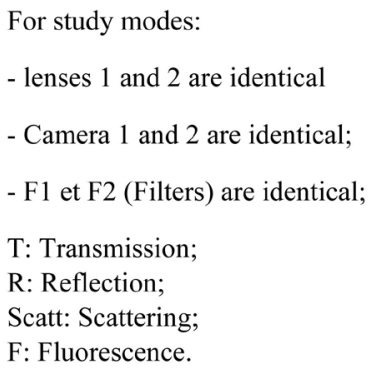

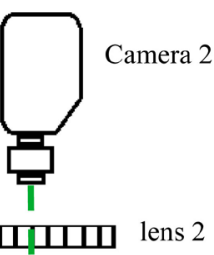

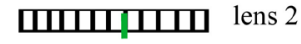
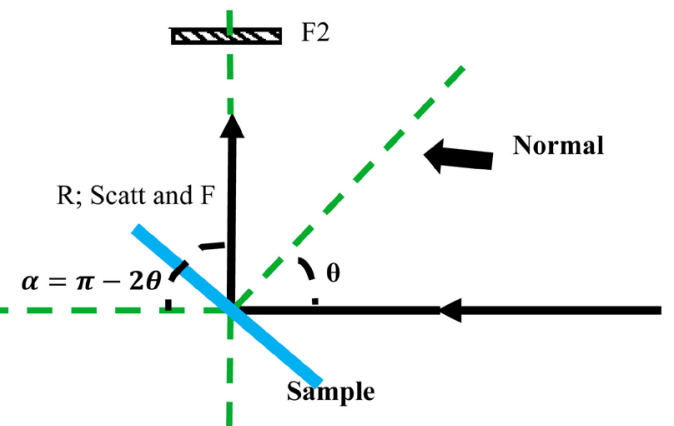

I

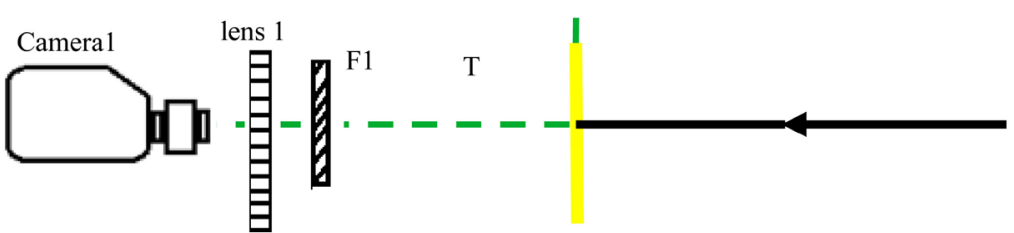

Figure 1. Diagram determining the angles of rotation for sample and camera in transmission, reflection, diffusion and/or fluorescence.

Table 1. The values of the angles of incidence and the rotation stages for transmission, reflection, scattering and fluorescence mode.

\begin{tabular}{ccc}
\hline Mode & $\theta$. Angle of incidence & $\begin{array}{c}\alpha \text { : Direction of the camera axis } \\
\text { by a report incidence }\end{array}$ \\
\hline Transmission & $0^{\circ}$ & $0^{\circ}$ \\
\hline Reflection & $30^{\circ}$ & $120^{\circ}$ \\
& $45^{\circ}$ & $90^{\circ}$ \\
& $50^{\circ}$ & $80^{\circ}$ \\
Scattering & $60^{\circ}$ & $60^{\circ}$ \\
\hline Fluorescence & $45^{\circ}$ & $90^{\circ}$ \\
\hline
\end{tabular}

photography. The designed tool consists to illuminate biological objects (diseased or healthy leaf plants) using an illumination source (either lasers or LEDs) to pass visible light through the lens in order to converge the incident flux towards sample. In fluorescence mode, a filter cuts the excitation light by recovering only the light emitted by the sample at the wavelength fluorescence retained. Finally, the high magnification objective allows an intermediate (enlarged) view of the sample captured by a CMOS camera, the image of which will be acquired using a computer.

Given that each biological sample has its optical properties, for better use of the optical system, it is also necessary to take into account the working wave- 
lengths and the selection of the angles for each mode of study.

Image acquisition has been validated on a few samples of plant stems and on a lettuce leaf (See the figures in the results section) to lead to a complete study within the framework of this research work.

\section{Initial Results}

The various stages of design of the optical system according to the modes of studies in transmission, reflection, diffusion and fluorescence are represented below in Figure 2 and Figure 3 with indication of the propagation of their light radiation, their angle of incidence and their direction of the axis of the camera.

Following the realization of this scientific work, after having familiarizing ourselves with the design of the new material, the operating principle of the device was validated on samples of plant stem and lettuce leaf in order to acquire their microscopic images according to the corresponding modes such as transmission, reflection, diffusion and fluorescence (Figure 4).

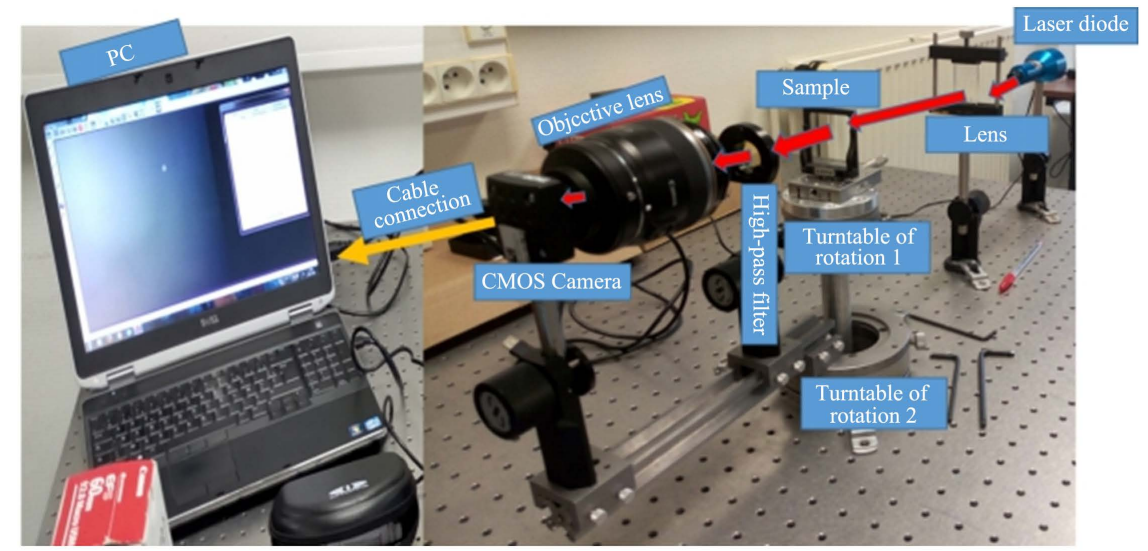

Figure 2. Design of the optical system in transmission mode with angle of incidence and direction of the axis of the camera: zero degree.

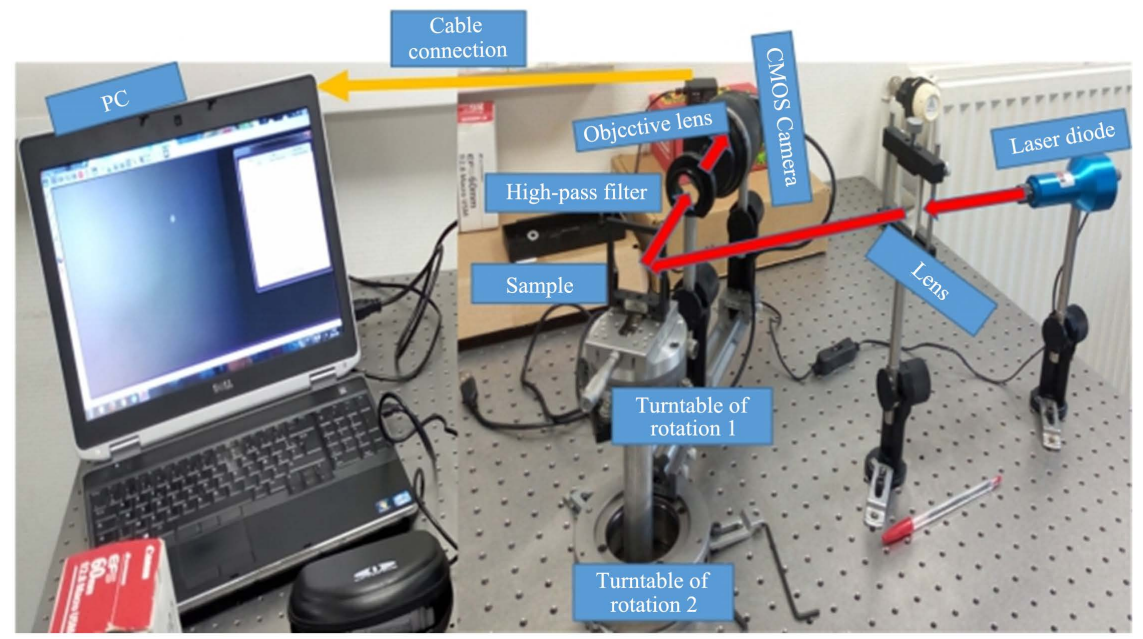

Figure 3. Design of the optical system in reflection, scattering and fluorescence mode with angle of incidence ( 45 degrees) and direction of the axis of the camera ( 90 degrees). 


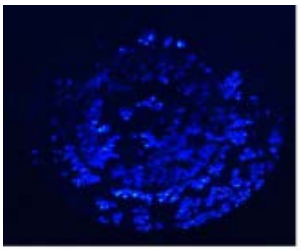

(a)

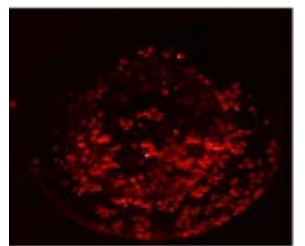

(d)

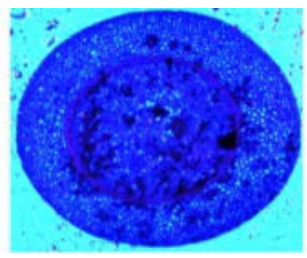

(b)

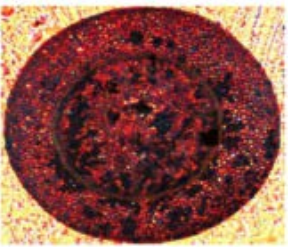

(e)

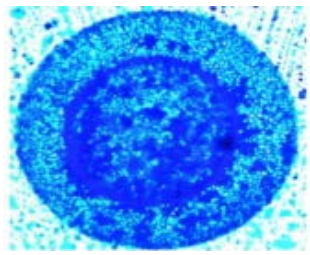

(c)

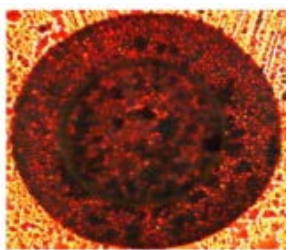

(f)

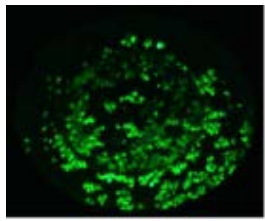

(g)

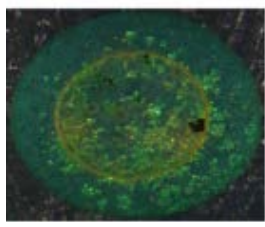

(k)

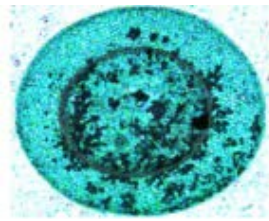

(h)

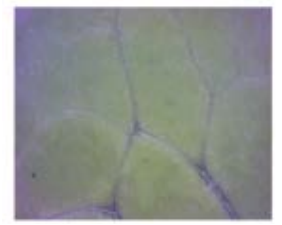

(l)

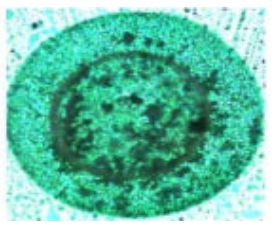

(i)

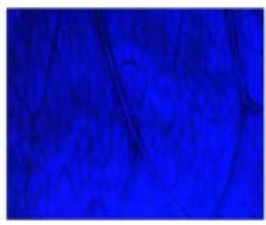

(m)

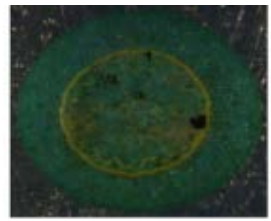

(j)

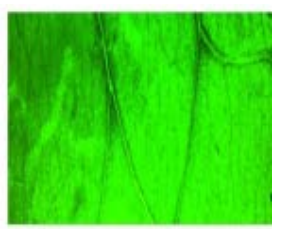

(n)

Figure 4. Example of microscopic images with some plant samples according to the corresponding modes ((a), (b), (c), (d), (e), (f), (g), (h), (i), (j), (k), (l), (m) and (n)). (a) Blue LED plant stem diffusion; (b) Blue LED plant stem transmission; (c) Tige plante LED bleu transmission filtre; (d) Tige plante LED rouge diffusion; (e) Tige plante LED rouge transmission; (f) Tige plante LED rouge transmission filtre; (g) Tige plante LED verte diffusion; (h) Tige plante LED vert transmission; (i) Tige plante LED vert transmission filtre; (j) Tige plante sans ecairage diffusion; (k) Tige plante ecairage avec neon diffusion; (l) Laitar ecairage ambiaet; (m) Laitue eacre flue LED blease; (n) Laitue eacre flue LED verte.

These series of microscopic and multimodal images will be used to reconstruct the corresponding spectrum wavelength by wavelength using the technique of optical and multimodal spectroscopy for a complementary study in order to have information on the state of health of the pathogens likely to attack crop plants [11] [12] [13]. In other fields such as biology for example, these types of image qualities can provide spectral information on the molecular biology processes existing in plant leaves or viral and bacterial morphological study of pathogens. Our ambition is to introduce inexpensive processes for in-the-field monitoring of the spread of plant diseases to improve agricultural yields. For most researchers, fluorescence images can also be useful in detecting plant cells which cannot be discriminated in transmission or other modes of study. In order to understand the epidemiology of a parasitic disease, it is necessary to iden- 
tify the causative agent and to study all the factors governing its development. This is how in the context of spectroscopy/microscopy applications to phytopathology, the evolution and especially the early detection of pathogens such as viruses and bacteria on diseased leaves is currently a research subject using spectroscopic and multimodal imaging data that can be exploited by statistical techniques. To better understand the performance of the recent imaging system, it is preferable to carry out the spectral and multimodal study of certain pathogens and their interaction in environments where plants are cultivated [11] [12] [13].

\section{Discussion}

This research work mainly aims to design a multimodal optical system with light sheet usable in transmission, reflection, diffusion and fluorescence modes for application in several fields of phytopathology in order to study the potentialities of the macro-imaging device derived from a work by David Rousseau, professor at the University of Angers, for "light sheets" imagery [22]; with a view to proposing a reference protocol defining the angles of rotation of the turntables of the camera and of the sample. The positive results of this research demonstrate that the main objectives have been achieved and will serve as a starting point for future work. This scientific research is on the one hand a complement of training by an initiation to the design of optical instruments, a subject which is not taught in Mali, and on the other hand the primer, on an imagery mounting concrete and partially innovative, of the reflection at the base of his future research subject in Bamako and almost elsewhere.

Furthermore, transmission, reflection, scattering and fluorescence studies were carried out by Swedish physicists [1] [2] [4], within the framework of cooperative research projects; using high quality microscopes better suited for microbiology than for plant pathology and which pose almost insoluble maintenance problems in some African environments. In addition, the lighting of leaf samples are made by light-emitting diodes; and these are limited to transparent samples in order to acquire in terms of qualities their multimodal image, finally they are exploitable for the application of optical spectroscopy [11] [12] [13]. However, these light emitting diodes are not intended for all types of plant leaf samples for microscopic and multimodal imaging. On the other hand, for the phytopathology study, the optical system carried out in this work is requested with any light source and regardless of sample properties for microscopic and multimodal imaging.

At the initiation of this work, the less sufficient available data did not prevent the realization of the instrument by association of some optical elements destined for the characterization of the spectral behavior of the phytopathological diseases proceeding to their follow-up in the fields of real-time plant cultures.

However, contacts with the agricultural community may exist through biology and may provide a selection of subjects for study. The hope is obviously in the long term to be able to introduce in full scale into agricultural holdings dedicated instruments useful for diagnosis and if possible for elimination certain para- 
sites and diseases of cultivated plants. The adaptable instrument is developed for many cases, easy both to use and keep in working order.

The economic importance can be considerable for the agro-food sector, justifying the implementation of research and development work to improve the tools for characterizing these pathogens and to acquire knowledge about their epidemiology to develop new control methods using the system designed for the plant pathology approach by optical and multimodal spectroscopy. The preventive measures adopted by microbiologists and agronomists in Mali and elsewhere require a complementary study based on optical and multimodal spectroscopy by improving the techniques of spectral characterization of plant diseases, in the agricultural field while developing the relative knowledge to minimize the propagation of these phytopathological diseases such as bacterial and viral diseases [15] [16] [23].

As a result, the designed and innovative optical system is capable of diagnosing pathogenic diseases that are poorly controlled because of their abundance and seasonal resistance in crop fields.

The design of this material will help the General Council of Agriculture to take decisions for the development of the agrarian pole in disadvantaged regions. Thus, the development of this multimodal imaging system for plants seems the most relevant than the biochemical interpretation systems to detect and/or study the effects of pathogens allowing physicists to definitively adopt the design of optical tools for phytopathology.

\section{Conclusion}

Regarding this research work based on the design of a multimodal optical system for the study of phytopathology, the results answer to the initial objectives. The interest of this project concerns the potential of the innovated instrument and these uses at different scales, namely the relevance of this work for Mali, mainly contributes to the establishment of an optical device capable of solving spectral diagnostic problems applied in any field. In addition, universities, colleges and institutes for agricultural engineers in the country will benefit from application support within the framework of laboratory work for the satisfaction of populations working in the agricultural sector. Thus, this research work is relevant and innovative, meets the needs of players in the applied physical sector at the national and international level.

In the field, crude and robust instruments will therefore have to be developed for each case selected, so that they can be deployed with very modest financial and human resources. For example, it will be necessary to limit oneself to minor modifications on mobile phone cameras, fitted with mounts to ensure illumination (in spectrum and orientation) and detection (at the desired magnification) under conditions specified in advance and reproducible.

\section{Acknowledgements}

A sincere thank addresses the Higher Normal School of Bamako for its adminis- 
trative support in taking advantage of this research project. The French Embassy is financially thanked for the opportunity of this quality and ambitious scientific work to develop the partnership between the University of Bamako and French laboratories.

\section{Conflicts of Interest}

The authors declare no conflicts of interest regarding the publication of this paper.

\section{References}

[1] Brydegaard, M., Guan, Z. and Svanberg, S. (2009) Broad-Band Multispectral Microscope for Imaging Transmission Spectroscopy Employing an Array of Light-Emitting Diodes. American Journal of Physics, 77, 104-110. https://doi.org/10.1119/1.3027270

[2] Brydegaard, M., Merdasa, A., Jayaweera, H., Alebring, J. and Svanberg, S. (2011) Versatile Multispectral Microscope Based on Light Emitting Diodes. Review of Scientific Instruments, 82, Article ID: 123106. https://doi.org/10.1063/1.3660810

[3] Zoueu, J.T., Ouattara, S., Toure, A. and Zan, S.T. (2009) Spectroscopic Approach of Multispectral Imaging of Plasmodium falciparum Infected Human Erythrocytes. IEEE Proceedings, ICTON Mediterranean Winter Conference, Vol. 23, 1-7. https://doi.org/10.1109/ICTONMW.2009.5385598

[4] Merdasa, A.J., Brydegaard, M., Svanberg, S. and Zoueu, J.T. (2013) Staining-Free Malaria Diagnostics by Multispectral and Multimodality Light-Emitting-Diode Microscopy. Journal of Biomedical Optics, Vol.18 (03): 036002.

https://doi.org/10.1117/1.JBO.18.3.036002

[5] Opoku-Ansah, J. anderson, B., Eghan, J.M., Boampong, J.N., Osei-Wusu Adueming, P., Amuah, C.L.Y. and Akyea, A.G. (2013) Automated Protocol for Counting Malaria Parasites ( $P$. falciparum) from Digital Microscopic Image Based on $L^{\star} a^{\star} b^{\star}$ Colour Model and KMeans Clustering. International Journal of Computer Science and Security, 7, 149-158.

[6] Bagui, K.O. and Zoueu, J.T. (2014) Red Blood Cells Counting by Circular Hough Transform Using Multispectral Images. Journal of Applied Sciences, 14, 3591-3594. https://doi.org/10.3923/jas.2014.3591.3594

[7] Opoku-Ansah, J., Eghan, J.M., Anderson, B. and Boampong, J.N. (2014) Wavelength Markers for Malaria (Plasmodium falciparum) Infected and Uninfected Red Blood Cells for Ring and Trophozoite Stages. Applied Physics Research, 6, 47-55. https://doi.org/10.5539/apr.v6n2p47

[8] Bagui, K.O., Yavo, W., Tano, D. and Zoueu, J.T. (2014) Etude de l'effet de l'amodiaquine sur les globules rouges infectés par le paludisme dans les images multi-spectrales. Afrique Science, 10, 36-44.

[9] Omucheni, D.L., Kaduki, K.A., Bulimo, W.D. and Angeyo, H.K. (2014) Application of Principal Component Analysis to Multispectral Multimodal Optical Image Analysis for Malaria Diagnostics. Malaria Journal, 13, Article No. 485.

http://www.malariajournal.com/content/13/1/485 https://doi.org/10.1186/1475-2875-13-485

[10] Bagui, K.O., Zoueu, J.T. and Wählby, C. (2015) Automatic Malaria Diagnosis by the Use of Multispectral Contrast Imaging. Journal of Physical Chemical News, 75, 86-98. 
[11] Sangare, M., Bagui, O.K., Traore, I., Babana, A.H., Ba, A. and Zoueu, J.T. (2015) Discrimination de différentes sous phénotypes du Ralstonia solanacearum dans une feuille de tabac par imagerie multi-spectrale. Afrique Science, 11, 95-103. http://www.afriquescience.info

[12] Sangare, M., Agneroh, T.A., Bagui, O.K., Traore, I., Ba, A. and Zoueu, J.T. (2015) Classification of African Mosaic Virus Infected Cassava Leaves by the Use of Multi-Spectral Imaging. Optics and Photonics Journal, 5, 261-272.

https://doi.org/10.4236/opj.2015.58025

http://www.scirp.org/journal/opj

[13] Sangare, M., Tekete, C., Bagui, O.K., Ba, A. and Zoueu, J.T. (2015) Identification of Bacterial Diseases in Rice Plants Leaves by the Use of Spectroscopic Imaging. Applied Physics Research Journal, 5, 61-69. https://doi.org/10.5539/apr.v7n6p61

[14] Kouakou, A.K., Soro, A.P., Taky, A.K., Patrice, K. and Zoueu, J.T. (2017) Multi-Spectral and Fluorescence Imaging in Prevention of Overdose of Herbicides: The Case of Maize. Spectral Analysis Reviews, 5, 11-24.

http://www.scirp.org/journal/sar https://doi.org/10.4236/sar.2017.52002

[15] Wonni, I., Cottyn, B., Detemmerman, L., Dao, S., Ouedraogo, L., Sarra, S., Tekete, C., Poussier, S., Corral, R., Triplett, L., Koita, O., Koebnik, R., Leach, J., Szurek, B., Maes, M. and Verdier, V. (2014) Analysis of Xanthomonas oryzae pv. oryzicola Population in Mali and Burkina Faso Eveals a High Level of Genetic and Pathogenic Diversity. Phytopathology, 104, 520-531.

https://doi.org/10.1094/PHYTO-07-13-0213-R

[16] Yadessa, G.B., Bruggen Van, A.H.C. and Ocho, F.L. (2010) Effects of Different Soil Amendments on Bacterial Wilt Caused by Ralstonia solanacearum and on the Yield of Tomato. Journal of Plant Pathology, 92, 439-450.

[17] Michel, V.V., Wang, J.F., Midmore, D.J. and Hartman, G.L. (1997) Effects of Intercropping and Soil Amendment with Urea and Calcium Oxide on the Incidence of Bacterial Wilt of Tomato and Survival of Soil-Borne Pseudomonas solanacearum in Taiwan. Plant Pathology, 46, 600-610. https://doi.org/10.1046/j.1365-3059.1997.d01-45.x

[18] Konaté, I. (2007) Diversité Phénotypique et Moléculaire du Caroubier (Ceratonia siliqua L.) et des Bactéries Endophytes qui lui sont Associées. These de Doctorat, Universite Mohammed V-Agdal Faculte des Sciences Rabat, 2-168.

[19] Saponari, M., Manjunath, K. and Yokomi, R.K. (2008) Quantitative Detection of Citrus tristeza Virus in Citrus and Aphids by Real-Time Reverse Transcription-PCR (TaqMan ${ }^{\circledR}$ ). Journal of Virological Methods, 147, 43-53.

https://doi.org/10.1016/j.jviromet.2007.07.026

[20] Ruiz-Ruiz, S., Ambros, S., Vives, M.D.C., Navarro, L., Moreno, P. and Guerri, J. (2009) Detection and Quantification of Citrus Leaf Blotch Virus by Taq Man Real-Time RTPCR. Journal of Virological Methods, 160, 57-62.

https://doi.org/10.1016/j.jviromet.2009.04.012

[21] Marcel, A.A., Zoueu, J.T. and Konan, K. (2016) Characterization of a Multimodal and Multispectral Led Imager: Application to Organic Polymer's Microspheres with Diameter $\Phi=10.2 \mu \mathrm{m}$. Optics and Photonics Journal, 6, 171-183.

http://www.scirp.org/journal/opj https://doi.org/10.4236/opj.2016.67019

[22] Rousseau, D. (2017) Microscopie 3D de fluorescence à feuillet de lumière. Vol. 111. Union des professeurs de physique et de chimie, 987-997. 
[23] Bigirimana, S. and Legg, J.P. (2007) La menace de la pandemie de la mosaique du manioc sur la production et ses consequences au Burundi. Proceedings of the 13 th ISTRC Symposium, Arusha, 359-364. 